\title{
2D Cu-TCNQ Metal-Organic Networks Induced by Surface Alloying
}

\author{
J. D. Fuhr, ${ }^{\dagger, \dagger}$ L. I. Robino, ${ }^{\ddagger}$ L. M. Rodríguez, ${ }^{\dagger}$ A. Verdini, ${ }^{\S}{ }^{\circledR}$ L. Floreano, ${ }^{\S}$ H. Ascolani, ${ }^{*}, \oplus$ \\ and J. E. Gayone* ${ }^{*},+\neq$ \\ ${ }^{\dagger}$ Centro Atómico Bariloche, CNEA y CONICET, Av. E. Bustillo 9500, R8402AGP Bariloche, Argentina \\ ${ }^{*}$ Instituto Balseiro, CNEA y Universidad Nacional de Cuyo, Av. E. Bustillo 9500. R8402AGP Bariloche, Argentina \\ ${ }^{\S}$ CNR-IOM, Laboratorio TASC, Basovizza SS14 Km. 163.5, I-34149 Trieste, Italy
}

Supporting Information

\begin{abstract}
We have studied the self-assembly of 7,7,8,8-tetracyanoquinodimethane molecules on the $(3 \sqrt{2} \times \sqrt{2}) R 45^{\circ}$ reconstruction of the $\mathrm{SnCu}(001)$ surface alloy by means of X-ray photoemission spectroscopy, scanning tunneling microscopy, near-edge X-ray absorption fine structure spectroscopy, and density functional theory calculations. Our results show that surface alloying strongly attenuates the chemical interaction of the molecule with the surface, but it does not inhibit the charge transfer from the substrate to the molecules. The assembly mechanism of the molecules is completely modified with respect to the bare $\mathrm{Cu}(001)$ surface. We show that, on the $\mathrm{SnCu}(100)$ surface alloy, the strong $\mathrm{CN}-\mathrm{Cu}$
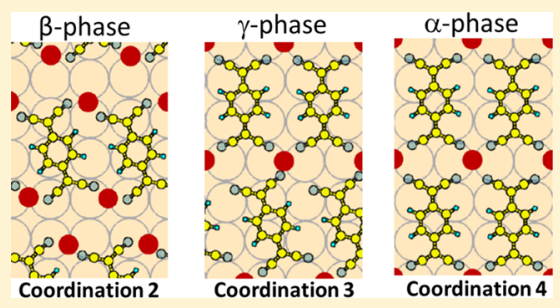
interaction drives the formation of different coordination structures with native $\mathrm{Cu}$ adatoms. We found that the flexible coordination chemistry of $\mathrm{Cu}$ allows the formation of three different stable phases, each one with the $\mathrm{Cu}$ ions in a different coordination geometry (coordinations 4,3 , and 2 ). Moreover, we show that both the formation of lateral $\mathrm{H}$ bonds between adjacent molecules and the interaction of the $\mathrm{Cu}$ ion with the substrate play determinant roles in the stabilization of the structures.
\end{abstract}

\section{INTRODUCTION}

The synthesis of two-dimensional (2D) supramolecular networks by the self-assembly of organic molecules on metal surfaces has attracted considerable attention for the possibility of conferring novel properties to a substrate. ${ }^{1-3}$ However, a rational production of these networks is still a difficult task since the stabilization and the functionality of the organic layer crucially depend on the interplay between the moleculemolecule interactions and the molecule-surface interactions ${ }^{4,5}$ Therefore, an appropriate control of the surface influence on the assembling mechanisms is required to build up and tune the properties of the organic layers. In this sense, much effort has been dedicated to study and compare the growth of supramolecular networks on metal surfaces with different degrees of reactivity.

Less explored substrates for the growth of supramolecular networks are the surface alloys, which have been extensively used in the study of heterogeneous catalysis. The deposition at room temperature (RT) of $0.5 \mathrm{ML}$ of $\mathrm{Sn}$ atoms on $\mathrm{Cu}(001)$ produces a single-layer surface alloy with a $(3 \sqrt{ } 2 \times \sqrt{ } 2) R 45^{\circ}$ reconstruction (in short $3 \sqrt{ } 2$ ). The corresponding $\mathrm{Sn} / \mathrm{Cu}$ composition is $3: 2$ due to the formation of $\mathrm{Cu}$ vacancy rows oriented along the [010] directions, ${ }^{6,7}$ as indicated in Figure $1 \mathrm{~b}$. At $360 \mathrm{~K}$, there is a reversible phase transition to a $(\sqrt{2} \times$ $\sqrt{ } 2) R 45^{\circ}$ reconstruction, where the $\mathrm{Cu}$ vacancies break their alignment by lateral displacement, whereas the $\mathrm{Sn}$ atoms remain, on average, close to the low-temperature positions. ${ }^{8,9}$ The $3 \sqrt{2}$ reconstruction is a promising system for fine-tuning the molecule/substrate interaction since $\mathrm{Sn}$ alloying signifi-

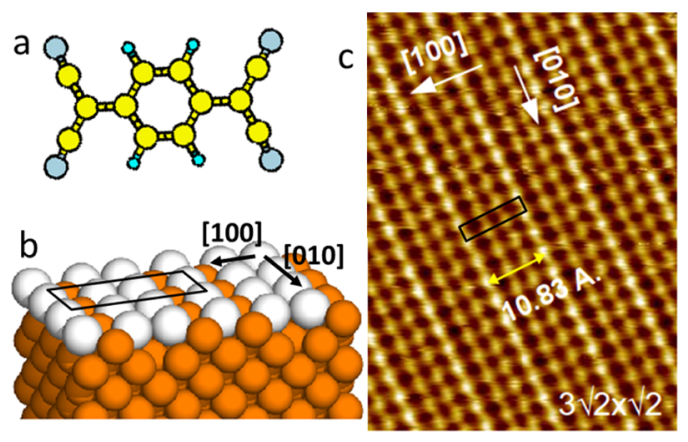

Figure 1. (a) 7,7,8,8-Tetracyanoquinodimethane (TCNQ) molecule. Yellow, C; light-blue, H; gray, N. (b) Schematic drawings of the $(3 \sqrt{ } 2 \times \sqrt{2}) R 45^{\circ}$ surface alloy reconstruction. White-gray (orange) corresponds to $\mathrm{Sn}(\mathrm{Cu})$ atoms. (c) Filled-state scanning tunneling microscopy $(\mathrm{STM})$ image of the $(3 \sqrt{2} \times \sqrt{2}) R 45^{\circ}$ reconstruction $(-0.05 \mathrm{~V}, 2 \mathrm{nA})$. Only the $\mathrm{Sn}$ atoms can be seen in the image.

cantly reduces the $\mathrm{Cu}(001)$ surface reactivity to carboxyl ${ }^{10,11}$ and amino groups. ${ }^{12}$

In this work, we investigate the response of the $3 \sqrt{ } 2$ surface alloy reconstruction to a reactive molecule, the strong organicacceptor 7,7,8,8-tetracyanoquinodimethane (TCNQ) molecule $^{13}$ (Figure 1a), and how the modified substrate affects the corresponding assembly of the molecules. Many studies

Received: September 4, 2019

Revised: December 3, 2019

Published: December 3, 2019 
regarding the assembly of TCNQ on different metallic crystalline surfaces have shown that phenomena such as electronic charge transfer and molecular bending strongly depend on the nature of the substrate (structure and electronics). ${ }^{14-20}$

Additionally, TCNQ has been extensively used as an organic ligand in the production of 2D metal-organic networks (MONs) on surfaces with different magnetic, electronic, and structural properties, which are also strongly affected by the choice of the substrate. ${ }^{21-28}$ In the previous reports, the molecules and the metal centers were separately evaporated on the surface. An alternative route to produce MONs is to incorporate atoms or to capture native adatoms directly from the substrate. The coordination to native adatoms has been proposed in a very recent re-evaluation of the TCNQ network stabilization on $\operatorname{Ag}(111)$, indeed. ${ }^{20}$ No MONs with native $\mathrm{Cu}$ atoms were reported for the TCNQ networks on $\mathrm{Cu}(001)^{15}$ and $\mathrm{Cu}(111),{ }^{9}$ although native adatoms on $\mathrm{Cu}$ crystalline surfaces are known to coordinate to the nitrile terminal groups of adsorbed molecules ${ }^{29-33}$

We show here that the modification of $\mathrm{Cu}(001)$ by $\mathrm{Sn}$ surface alloying strongly reduces the chemical interaction of TCNQ molecules with the surface, which completely modifies the self-assembly mechanisms and gives rise to three different coordination structures based on the incorporation of $\mathrm{Cu}$ adatoms.

\section{METHODS}

The experiments of scanning tunneling microscopy (STM) were carried out at the Centro Atómico Bariloche, Argentina, using an ultrahigh vacuum system from Omicron Nanotechnology. All of the STM images reported in this work were taken at RT using W tips. The chamber is equipped with a lowenergy electron diffraction (LEED) optics, a home-made sputtering gun, and evaporators. The $\mathrm{Cu}(001)$ substrate (Mateck, orientation accuracy $\pm 0.1^{\circ}$ ) was prepared by repeated cycles of $\mathrm{Ar}^{+}$at $1.5 \mathrm{keV}$ bombardment and then annealing at $770 \mathrm{~K}$. Sn was evaporated from a home-made resistively heated boron-nitride $(\mathrm{BN})$ crucible onto the clean $\mathrm{Cu}(001)$ surface, kept at $\mathrm{RT}$, at a rate of 0.1 monolayer $(\mathrm{ML})$ every $2.5 \mathrm{~min}$. The formation of the $3 \sqrt{2}$ structure was checked by LEED. The TCNQ molecules (Sigma-Aldrich, 98\% purity) were also evaporated from a home-made resistively heated $\mathrm{BN}$ crucible at a rate of approximately 0.1 ML every $5 \mathrm{~min}$. We define $1 \mathrm{ML}$ as the coverage corresponding to 1 molecule or atom per $\mathrm{Cu}$ atom of the top $\mathrm{Cu}(001)$ surface. All of the TCNQ adsorption were done with the surface alloy $(3 \sqrt{2}$ reconstruction) kept at a fixed $T$. Note that when $T$ is above $360 \mathrm{~K}$, the reconstruction is $(\sqrt{2} \times$ $\sqrt{ } 2) R 45^{\circ}$, as indicated in the Introduction section.

The X-ray photoemission spectroscopy (XPS) and nearedge X-ray absorption fine structure spectroscopy (NEXAFS) experiments were carried out at the ALOISA beamline ${ }^{34,35}$ of the ELETTRA synchrotron, at Trieste. The XPS spectra were measured at grazing incidence ( $4^{\circ}$ from the surface) and in normal emission by means of a hemispherical electron analyzer (angular acceptance of $2^{\circ}$ ). We measured the $\mathrm{N} \mathrm{1s,} \mathrm{Sn} \mathrm{4d,} \mathrm{and}$ $\mathrm{Cu} 3 \mathrm{p}$ photoemission spectra with a photon energy of $515 \mathrm{eV}$ and an overall energy resolution of $230 \mathrm{meV}$. We also measured the $\mathrm{C} 1 \mathrm{~s}$ and $\mathrm{Cu} 3 \mathrm{p}$ with a photon energy of $380 \mathrm{eV}$ (overall resolution of $140 \mathrm{meV}$ ). The binding energy (BE) of each spectrum is calibrated to the position of the $\mathrm{Cu} 3 \mathrm{p}$ peak. NEXAFS spectra at the nitrogen K-edge were taken by means of partial electron yield with a channeltron facing the sample and a negatively biased grid in between for rejecting lowenergy secondary electrons (bias of $-370 \mathrm{~V}$ ). The photon energy resolution was set to about $100 \mathrm{meV}$. Absolute energy calibration has been obtained a posteriori through the nitrogen absorption signal observed in the $I_{0}$ drain current, as measured on the metal coating of the refocusing mirror, that was independently calibrated by simultaneous acquisition of the gas phase absorption spectra from $\mathrm{N}_{2} \cdot{ }^{35}$ The spectra were collected by keeping the sample at constant grazing angle of 6 degrees with respect to the surface while rotating the surface around the photon beam axis to switch from transverse magnetic ("p") to transverse electric ("s") polarization. Further details about the scattering geometry can be found in ref 36 . To compensate for the variation of the photon flux across the $\mathrm{N}$ K-edge, we have normalized the NEXAFS spectra from the TCNQ overlayer to the spectra taken from the bare $3 \sqrt{ } 2$ reconstruction.

We performed the calculations based on the density functional theory (DFT) using the Quantum ESPRESSO package, ${ }^{37}$ a plane wave implementation with the projected augmented wave method. To take into account possible molecule-surface van der Waals interactions, we used the rVV10 exchange-correlation functional, ${ }^{38}$ which is a revised version of VV10 nonlocal density functional by Vydrov and Van Voorhis. ${ }^{39}$ For the surface calculations, we have used the slab method with five $\mathrm{Cu}$ layers and the $\mathrm{Sn}$ alloy in the topmost layer. For all of the calculations, we fixed the two lower layers, while all other atoms are allowed to relax. We used a wave function/charge cutoff of 60/600 Ry, and Brillouin integrations were done using a grid equivalent to $12 \times 12 \times 1 k$-points for the surface unit cell.

To compare the energy of different molecular configurations, we evaluated a weighted molecular adsorption energy, $\epsilon_{\text {ads }}$, for each configuration as

$$
\epsilon_{\mathrm{ads}}=-\left[\frac{E_{\mathrm{conf}}-E_{\text {surf }}-N_{\mathrm{at}} E_{\mathrm{at}}}{N_{\mathrm{TCNQ}}}-E_{\mathrm{TCNQ}}\right]
$$

where $E_{\text {conf }}$ is the total energy of the configuration of $N_{\text {TCNQ }}$ molecules adsorbed on the surface with $N_{\text {at }}$ adatoms, $E_{\text {surf }}$ is the total energy of the clean surface with the same area, and $E_{\mathrm{TCN} Q}$ is the total energy of an isolated (gas phase) molecule. In this experiment, all of the metal atoms that are part of the coordination structures are not codeposited with the molecules but are directly incorporated from the substrate either by metal extraction (like found for most of porphyrinoids on metals and oxides $^{40,41}$ ) or by capture of native adatoms. The latter ones are formed by atom detachment at kinks, where the corresponding energy cost for attachment/detachment is identical to that of a bulk energy atom. ${ }^{42}$ Therefore, for configurations with adatoms $(\mathrm{Cu}$ or $\mathrm{Sn}), E_{\mathrm{at}}$ is the reference energy per atom: for $\mathrm{Cu}$, we have taken the corresponding bulk energy, and for $\mathrm{Sn}$, we have taken the energy of an Sn atom in the $3 \sqrt{2}$ surface alloy reconstruction.

\section{RESULTS AND DISCUSSION}

The assembly of TCNQ on $\mathrm{Cu}(001)$ at room temperature (RT) has been studied by Tseng et al.. ${ }^{15}$ In this system, there is a considerable electronic charge transfer from the surface to the molecule. The molecules arrange in rectangular-shape islands with a preferential growth direction, as indicated by the yellow arrow in Figure 2a. From the STM image, we obtained 


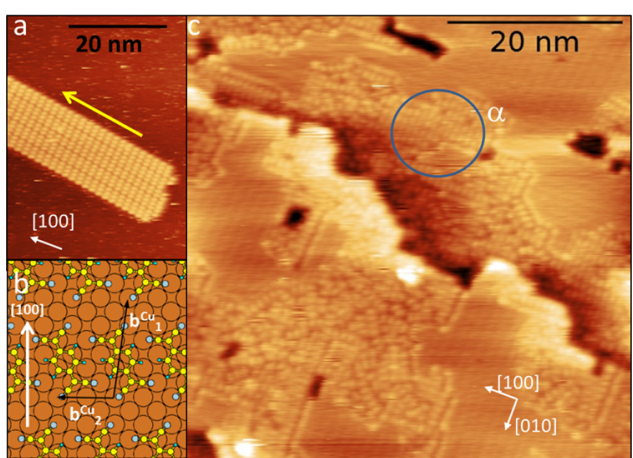

Figure 2. (a) STM image obtained after the adsorption of TCNQ molecules at RT on the clean $\mathrm{Cu}(001)(-2 \mathrm{~V}, 5 \mathrm{pA})$. The yellow arrow indicates the preferential growth direction. (b) Calculated relaxed structure of TCNQ on $\mathrm{Cu}(001)$. (c) Similar to (a) but for the adsorption on the $3 \sqrt{ } 2$ surface alloy reconstruction $(2 \mathrm{~V}, 5 \mathrm{pA})$. The ellipse highlights the $\alpha$ phase.

a unit cell of $b_{1}^{\mathrm{Cu}}=(12.5 \pm 1) \AA$ and $b_{2}^{\mathrm{Cu}}=(7.4 \pm 0.7) \AA$ and a subtended angle of $\alpha=98 \pm 3^{\circ}$ in agreement with previously reported values. ${ }^{15}$ In terms of the unit cell vectors $\mathbf{a}_{1}$ and $\mathbf{a}_{2}$ of the underlying $\mathrm{Cu}(001)$ substrate, the obtained unit cell vectors of the adlayer are compatible with a commensurate structure: $\mathbf{b}_{1}^{\mathrm{Cu}}=4 \mathbf{a}_{1}+3 \mathbf{a}_{2}$ and $\mathbf{b}_{2}^{\mathrm{Cu}}=-2 \mathbf{a}_{1}+2 \mathbf{a}_{2}$ (see Figure $2 \mathrm{~b}$ and SI-1). According to Tseng et al., ${ }^{15}$ there are not $\mathrm{Cu}$ adatoms in this structure but a strong interaction between the nitrile groups and the $\mathrm{Cu}$ atoms, causing rippling of the top surface layer. Remarkably, the molecular domains grow along a direction that corresponds to a head-to-head molecular alignment. Clearly, the molecule-to-substrate interaction overcomes also the electrostatic repulsion between the nitrile groups.

To compare with the surface alloy, we performed DFT calculations also for the $(43 \overline{2} 2)-\mathrm{TCNQ} / \mathrm{Cu}(100)$ phase. We obtained an adsorption energy $\left(\epsilon_{\mathrm{ads}}\right)$ of $3.68 \mathrm{eV}$ and a $\mathrm{N}-\mathrm{Cu}$ bond length of $d_{\mathrm{N}-\mathrm{Cu}}=2.05 \AA$ (Table 1). Additionally, the calculations indicate that the $\mathrm{CN}$ groups are bent toward the surface, forming an angle of $\phi=15.5^{\circ}$ with respect to the surface plane, in agreement with previous results ${ }^{15}$

Figure $2 c$ shows a large-scale STM image after RT deposition of TCNQ on the $3 \sqrt{2}$ surface alloy reconstruction. The parallel lines that are clearly distinguished in the areas of the surface free of molecules correspond to the $3 \sqrt{2}$ reconstruction and are oriented along the [100] and [010] crystallographic directions. Although the prepared interface shows no long-range order, a quasi-rectangular structure is observed at the local level, as indicated in Figure 2c ( $\alpha$ phase).

Figure 3a shows a domain of the $\alpha$ phase, where the rectangular unit cell $\left(b_{1}^{\alpha}=11 \pm 0.5 \AA\right.$ and $\left.b_{2}^{\alpha}=7.4 \pm 0.5 \AA\right)$ is indicated, compatible with a commensurate $(3 \sqrt{2} \times 2 \sqrt{2})$ $R 45^{\circ}$ unit cell and corresponding to a coverage of $1 / 12 \mathrm{ML}$. The small intermolecular distance of this arrangement implies that the negatively charged $\mathrm{CN}$ groups are facing one each other, which would be energetically unfavorable..$^{20,32,33}$ Additionally, the unit cell of this phase is very similar to that of the MONs formed by TCNQ with $\mathrm{Ni}^{23,25} \mathrm{Mn}^{24}$ and $\mathrm{Sn}^{28}$ as coordinating atoms on different substrates. We then concluded that the $\alpha$ phase is stabilized by a metal-ligand coordination interaction. DFT calculations show that coordination to $\mathrm{Cu}$ adatoms is more favorable from an energetic point of view than to Sn adatoms (see below). Therefore, we propose for the $\alpha$ phase the model shown in Figure 3b, where each $\mathrm{Cu}$ adatom is
Table 1. Adsorption Energy $\left(\epsilon_{\text {ads }}\right), \mathrm{N}-\mathrm{Cu}$ Bond Distance $\left(d_{\mathrm{N}-\mathrm{Cu}}\right), \mathrm{N}-\mathrm{Sn}$ Bond Distance $\left(d_{\mathrm{Sn}-\mathrm{Cu}}\right)$, and CN Angle, $\phi$, with Respect to the Surface Obtained from DFT Calculations for the Different Configurations Discussed in the Text ${ }^{a, b}$

\begin{tabular}{|c|c|c|c|c|}
\hline $\mathrm{Cu}(001)$ & $\epsilon_{\text {ads }}$ & $d_{\mathrm{N}-\mathrm{Cu}}$ & $d_{\mathrm{N}-\mathrm{Sn}}$ & $\phi$ \\
\hline$\left(\begin{array}{ll}4 & 3 \\
-2 & 2\end{array}\right)$ & $3.68 \mathrm{eV}$ & $2.05 \AA$ & & $15.5^{\circ}$ \\
\hline$(3 \sqrt{ } 2 \times \sqrt{ } 2) R 45^{\circ}$ & $\epsilon_{\mathrm{ads}}$ & $d_{\mathrm{N}-\mathrm{Cu}}$ & $d_{\mathrm{N}-\mathrm{Sn}}$ & $\phi$ \\
\hline$\alpha$ phase & $2.65 \mathrm{eV}$ & $2.1 \AA$ & & $6^{\circ}$ \\
\hline configuration (I) & $1.90 \mathrm{eV}$ & & $\begin{array}{r}2.79 \AA ; \\
2.97 \AA\end{array}$ & $\begin{array}{r}0.49^{\circ} ; \\
0.69^{\circ}\end{array}$ \\
\hline configuration (II) & $2.20 \mathrm{eV}$ & & $\begin{array}{l}2.48 \AA ̊ ; \\
\quad 3.73 \AA\end{array}$ & $14.6^{\circ} ; 1.3^{\circ}$ \\
\hline configuration (III) & $2.67 \mathrm{eV}$ & $1.97 \AA ̊$ & & $5.5^{\circ}$ \\
\hline $\begin{array}{l}\text { configuration (IV) } \beta \\
\text { phase }\end{array}$ & $2.71 \mathrm{eV}$ & $\begin{array}{l}2.02 \AA ; \\
1.96 \AA\end{array}$ & & $5.7^{\circ} ; 9.6^{\circ}$ \\
\hline $\begin{array}{l}\text { Sn-TCNQ single } \\
\text { stripe }\end{array}$ & $0.3 \mathrm{eV}$ & & $\begin{array}{r}2.37 \AA ; \\
2.4 \AA\end{array}$ & $\begin{array}{r}-9.50^{\circ} ; \\
-1.8^{\circ}\end{array}$ \\
\hline unsupported & & & $d_{\mathrm{N}-\mathrm{Cu}}$ & $d_{\mathrm{N}-\mathrm{Sn}}$ \\
\hline $\mathrm{Cu}-\mathrm{TCNQ}$, rectangul & alar & & & $0^{\circ}$ \\
\hline $\mathrm{Cu}-\mathrm{TCNQ}$, rotated & & & $\AA ̊ \AA 1.97 \AA ̊$ & $0^{\circ}$ \\
\hline
\end{tabular}

${ }^{a_{T}}$ Two values are indicated for the configurations with inequivalent $\mathrm{CN}$ groups. ${ }^{b}$ In the case of the unsupported configurations, the relative adsorption energy $\left(\Delta \epsilon_{\text {ads }}\right)$ is measured with respect to the rectangular one.
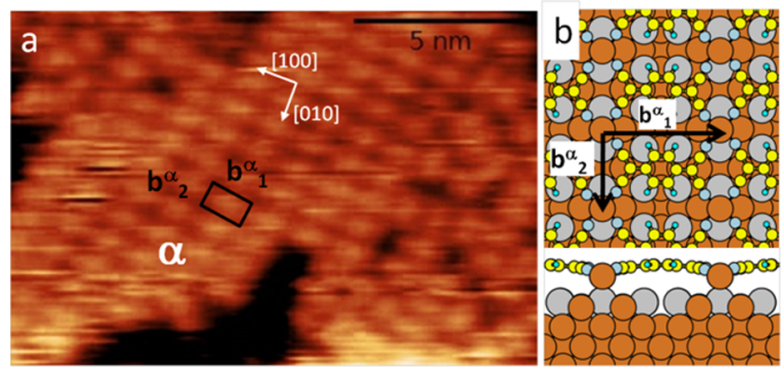

Figure 3. (a) STM image of a domain of the $\alpha$ phase obtained after depositing TCNQ on the $3 \sqrt{ } 2$ surface alloy reconstruction at $330 \mathrm{~K}$ $(2 \mathrm{~V}, 10 \mathrm{pA})$. (b) Calculated relaxed structure of the $\alpha$ phase model. White-gray (orange) corresponds to $\mathrm{Sn}(\mathrm{Cu})$ atoms.

coordinating four $\mathrm{CN}$ groups, with a $\mathrm{Cu} / \mathrm{TCNQ}$ stoichiometry ratio of $1: 1$. Figure $3 \mathrm{~b}$ actually corresponds to the relaxed structure obtained by DFT for the $\alpha$ phase. The calculations reveal that the $\mathrm{Cu}$ adatoms are in a bridge position between two Sn atoms and they lie in a plane $1.1 \AA$ below that of the molecular rings. In addition, the $\mathrm{CN}$ groups are bent toward the surface, forming an angle of $\phi=6^{\circ}$ with respect to the surface plane (Table 1). The $\mathrm{N}-\mathrm{Cu}$ bond distance is $2.1 \AA$, and the calculated adsorption energy is $\epsilon_{\text {ads }}=2.65 \mathrm{eV}$.

By increasing the substrate temperature to $420 \mathrm{~K}$ during molecular deposition, extended and ordered ribbon-shape domains of a new phase $(\beta)$ are developed along the [100] crystallographic direction (and the equivalent [010] one), whereas the $\alpha$ phase is no longer observed, as shown in Figure 4a. In Figure 4b, we show a zoomed image (empty states) where the molecular arrangement for the $\beta$ phase is seen in better detail. The proposed unit cell of the molecular layer, with $b_{1}^{\beta}=12.8 \pm 0.5 \AA, b_{2}^{\beta}=7.4 \pm 0.5 \AA$, and a subtended angle of $99 \pm 2^{\circ}$, is indicated in Figure $4 \mathrm{~b}$.

Interestingly, the STM image presents dark valleys between the molecular stripes and the interstripe distance $\left(d^{\beta}=12.7 \pm\right.$ 


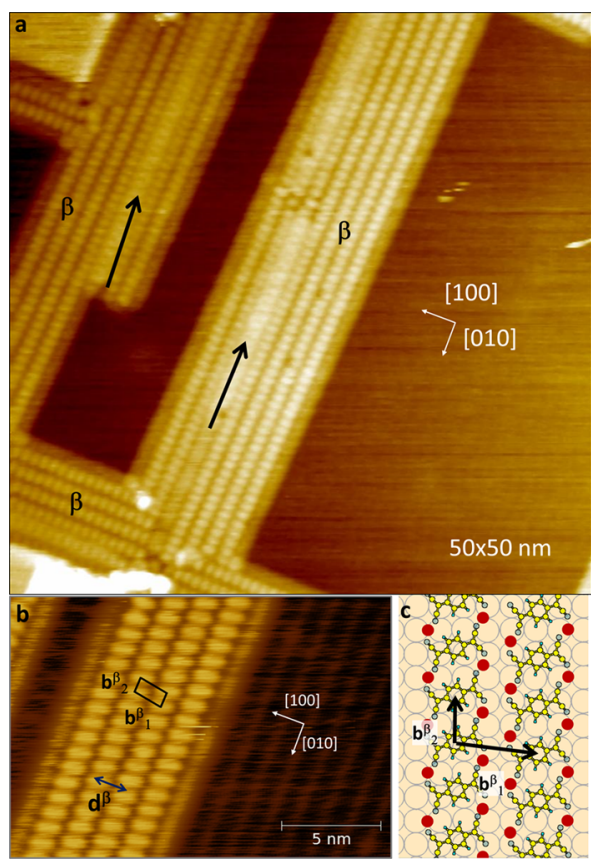

Figure 4. STM images obtained after the adsorption of TCNQ molecules at $420 \mathrm{~K}$ on the $3 \sqrt{2}$ surface alloy reconstruction. (a) Filled-state image $(-2 \mathrm{~V}, 5 \mathrm{pA})$ of the $\beta$ phase. The arrows indicate small domains of the $\gamma$ phase. (b) Empty-state image of the $\beta$ phase (2 $\mathrm{V}, 5 \mathrm{pA})$. The unit cell of the molecular layer and the interstripe distance, $d^{\beta}$, is depicted in the image. (c) Proposed model for the $\beta$ phase on the $3 \sqrt{2}$ reconstruction, where the red circles correspond to $\mathrm{Cu}$ adatoms. Only the $\mathrm{Sn}$ atoms (circles) of the substrate are shown.

$0.5 \AA$, Figure $4 \mathrm{~b})$ is significantly larger than in the $\alpha$ phase $\left(d^{\alpha}\right.$ $=b_{1}^{\alpha}=11 \AA$, Figure 3a). Compared with the $\alpha$ phase, the $\beta$ one is less dense with a molecular coverage of $1 / 14 \mathrm{ML}$. We therefore discard the possibility of an $\alpha$-like phase with $\mathrm{Cu}$ adatoms coordinating $\mathrm{CN}$ groups between molecules of different stripes. Figure $4 \mathrm{c}$ shows the model for the $\beta$ phase, as will be discussed hereafter, where each $\mathrm{Cu}$ adatom is coordinating two $\mathrm{CN}$ groups of adjacent molecules with stoichiometry $\mathrm{Cu}_{2} \mathrm{TCNQ}$. This model is compatible with a commensurate structure, where the $\mathrm{Cu}$ adatoms are in bridge positions between two $\mathrm{Sn}$ atoms, as in the case of the $\alpha$ phase (see SI-2 for more details).

We note that the unit cell shown in Figure $4 \mathrm{~b}$ is very similar to that formed by TCNQ adsorbed on $\mathrm{Cu}(100)$. However, there also are important differences between the two systems. The preferential growth direction of the $\beta$ phase is along the molecular stripes (short molecular axis) and, thus, is opposite to that observed on the bare substrate. Another difference is that temperatures above $400 \mathrm{~K}$ are needed to form ordered domains of the $\beta$ phase on the $\mathrm{SnCu}(001)$ surface alloy, while large domains of adsorbed TCNQ molecules are formed on the bare $\mathrm{Cu}(001)$ substrate at room temperature.

Taking into account the preferential growth direction we have performed calculations for different configurations corresponding to a single stripe of molecules using a $2 \sqrt{ } 2 \times$ $6 \sqrt{ } 2 R 45^{\circ}$ supercell (see Figure 5 and Table 1). A stripe formed by TCNQ molecules oriented with the long-axis perpendicular to the [010] direction (configuration I, Figure $5 \mathrm{a})$ is not energetically favorable having $\epsilon_{\text {ads }}=1.90 \mathrm{eV}$ compared to $\epsilon_{\text {ads }}=1.94 \mathrm{eV}$ for an isolated molecule (see SI-3 for extra details of the calculation).

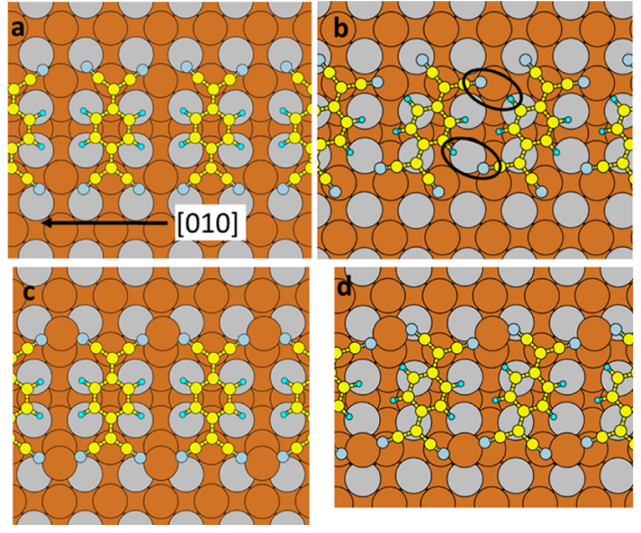

Figure 5. Configurations corresponding to a single stripe of TCNQ molecules adsorbed on the $\mathrm{SnCu}(001)$ surface alloy. (a) Molecules perpendicular in the [010] direction. (b) Rotated molecules with the formation of two $\mathrm{CH} \cdots \mathrm{N}$ bonds. (c) and (d) Similar to (a) and (b) but with $\mathrm{Cu}$ adatoms linking the TCNQ molecules. White-gray (orange) corresponds to $\mathrm{Sn}(\mathrm{Cu})$ atoms.

When the molecules are allowed to fully relax by in-plane molecular reorientation (configuration II, Figure 5b), the calculated adsorption energy is $\epsilon_{\text {ads }}=2.20 \mathrm{eV}$, which represents an energy gain per molecule of $0.3 \mathrm{eV}$ with respect to configuration I. This energy gain is associated with the formation of two $\mathrm{CH} \cdots \mathrm{N}$ bonds per molecule as indicated by the ellipses in Figure $5 \mathrm{~b}$. In this configuration, the $\mathrm{CN}$ groups that are forming $\mathrm{H}$ bonds are almost parallel to the surface plane $\left(\phi=1.3^{\circ}\right)$. The other ones are bent toward the surface by an angle $\phi=14.6^{\circ}$ (Table 1) and with a N-Sn bond distance of $2.48 \AA$. This distance is rather large for a covalent bond and reflects the low chemical interaction of the $\mathrm{CN}$ groups with the alloyed Sn layer. The adsorption energy of this configuration is lower than that obtained for the MON configuration associated with the $\alpha$ phase (see Table 1). It is worth mentioning that in this theoretical structure, which is free of $\mathrm{Cu}$ adatoms, the molecules are partially charged, as follows from the corresponding projected density of states (PDOS) where the lowest unoccupied molecular orbital (LUMO) is partially filled (see Figure 6a). According to the Bader analysis, ${ }^{43,44}$ the charge transfer from the surface to the molecules corresponds to $1.1 e^{-} /$molecule.
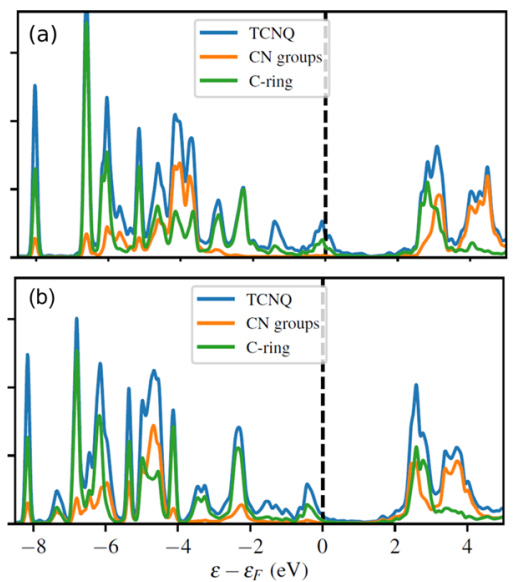

Figure 6. Projected density of states on the TCNQ molecules. (a) Configuration (II) and (b) configuration (IV). 
We then explore the same configurations but with $\mathrm{Cu}$ adatoms linking the TCNQ molecules: configurations III and IV in Figure $5 c, 5 d$, respectively. Note that configuration IV corresponds to a single stripe of the proposed model for the $\beta$ phase. The adsorption energies are rather similar for these two configurations: $\epsilon_{\mathrm{ads}}=2.67 \mathrm{eV}$ for the TCNQ molecules oriented perpendicular to the [010] direction (configuration III) and $\epsilon_{\mathrm{ads}}=2.71 \mathrm{eV}$ when they are allowed to relax by inplane rotation (configuration IV). Thus, the inclusion of $\mathrm{Cu}$ adatoms results in a considerable net energy gain per molecule and supports the $\mathrm{Cu}_{2} \mathrm{TCNQ}$ structure proposed for the $\beta$ phase (configuration IV). In addition, the calculated energy gain of $0.06 \mathrm{eV}$ for the single stripe of the $\beta$ phase with respect to the $\alpha$ one is in agreement with the STM images shown in Figure 4, where the latter phase is not observed. Moreover, the charge transfer to the molecules is higher than that obtained for the case without adatoms $\left(1.4 e^{-} /\right.$molecule, for configuration IV) and the PDOS (Figure 6b) shows that now the LUMO is completely filled. The fact that $\mathrm{Cu}$ adatoms are coordinating two adjacent $\mathrm{CN}$ groups explains the observed preferential growth direction along the stripes.

We point out that the same configuration but with $\mathrm{Sn}$ adatoms instead of $\mathrm{Cu}$ ones has an adsorption energy of only $\epsilon_{\text {ads }}=0.3 \mathrm{eV}$ (see Table 1 and SI-4). We have also compared different unsupported configurations for $\mathrm{Sn}_{2} \mathrm{TCNQ}$ and $\mathrm{Cu}_{2}$ TCNQ single stripes (see SI-5), and in all of the cases, there is an important energy gain for the coordination with $\mathrm{Cu}$ adatoms with respect to the Sn ones. In addition, it was recently shown that the interaction between nitrile ligands and $\mathrm{Cu}$ atoms is strong enough to prevent the formation of $\mathrm{Sn}$ TCNQ coordination networks on the $\mathrm{Cu}(001)$ surface. $^{28}$ Therefore, we discard the possibility of a Sn-TCNQ MON on the $3 \sqrt{ } 2$ surface alloy.

Coming back to configuration (IV) shown in Figure 5d, we note that the dicyanomethilene groups are slightly twisted so that the CN groups are not equivalent ((1) and (2) in SI-6). We obtain from the calculations two bond distances $d_{\mathrm{N}-\mathrm{Cu}}=$ $1.96 / 2.02 \AA$ for the $\mathrm{CN}$ groups (1) and (2), respectively, which are very close to the obtained value of the bare $\mathrm{Cu}(001)$ surface (see Table 1) and to previously reported values for $\mathrm{Cu}-\mathrm{CN}$ MONs in coordination $2^{30,32,45}$ In addition, the $\mathrm{Cu}$ adatoms lie in a plane at $1.6 \AA$ below the molecular rings, which is consistent with the fact that the $\mathrm{Cu}$ coordination adatoms were never detected by STM topography.

The intermediate position of the $\mathrm{Cu}$ adatoms between the Sn layer and the plane defined by the molecule rings reflects a considerable attractive interaction of $\mathrm{Cu}$ adatoms with the $\mathrm{Sn}$ layer. Indeed, the more stable configuration obtained for a single unsupported stripe of $\mathrm{Cu}_{2}$ TCNQ is one with the $\mathrm{Cu}$ adatoms at the same plane of the molecules rings (see SI-5). Pointing in the same direction, the calculated unsupported $\mathrm{Cu}_{2}$ TCNQ structure shows no in-plane rotation of the molecules as the one observed for configuration IV (Figure $5 d)$. Putting all together, we conclude that the energy gain made by the molecules in going from configuration III to IV $(0.04 \mathrm{eV})$ should be mainly driven by the interaction of the $\mathrm{Cu}$ adatoms with the substrate.

We point out that the comparison of the obtained adsorption energies reveals that alloying the $\mathrm{Cu}(001)$ surface with $\mathrm{Sn}$ strongly reduces the molecule-surface interaction. Indeed, configurations I and II shown in Figure 5 present a much lower adsorption energy with respect to the TCNQ/ $\mathrm{Cu}(001)$ system. The origin of this difference between the two substrates is found in the combination of two effects. In the case of the surface alloy, the $\mathrm{CN}$ groups can only interact with the $\mathrm{Sn}$ atoms, which protrude by $0.5 \AA$ above the $\mathrm{Cu}$ ones in the same alloy layer; hence, the $\mathrm{CN}$ groups cannot approach the $\mathrm{Cu}$ atoms underneath close enough to establish a chemical bond. On the other hand, the chemical interaction established between the $\mathrm{CN}$ groups and the $\mathrm{Cu}$ atoms of the $\mathrm{Cu}(001)$ surface is stronger than that established with the $\mathrm{Sn}$ atoms of the surface alloy, as follows from the comparison of the bond distances calculated for $\mathrm{N}-\mathrm{Cu}$ and $\mathrm{N}-\mathrm{Sn}$ (see Table 1). Overall, the higher chemical interaction of TCNQ nitrile terminations with $\mathrm{Cu}$ atoms rather than with $\mathrm{Sn}$ ones can be associated with the $3 \mathrm{~d}$ band (which drives the reactivity of transition metals $\left.{ }^{46}\right)$ in copper.

The production of MONs with atoms coming from the underlying substrate depends on the availability of adatoms, ${ }^{30}$ which usually increases with the surface temperature, and, in some cases, the molecule itself plays an active role and may favor the extraction of the atoms beneath. ${ }^{47}$ Along with the latter mechanism, we have calculated an energy barrier of $E_{\mathrm{b}}=$ $0.34 \mathrm{eV}$ for the creation of a $\mathrm{Cu}$ vacancy to form one TCNQcoordinated adatom when the molecules are already adsorbed on the surface (see SI-7 for the details of the calculation). Assuming a frequency factor of $\nu_{0}=10^{12} \mathrm{~s}^{-1}$ for this process, it gives a large rate for the transition for $T=298 \mathrm{~K}$ : $\nu=\nu_{0} \exp \left(-\frac{E_{\mathrm{b}}}{k_{\mathrm{b}} T}\right)=1.8 \times 10^{6} \mathrm{~s}^{-1}$, which assures the availability of $\mathrm{Cu}$ adatoms and is consistent with the formation of metal-organic structures with $\mathrm{Cu}$ already at RT.

A careful inspection of the STM image of Figure 4a shows that there are molecules with different apparent heights (black arrows). These molecules form a third phase that we named $\gamma$. Increasing the coverage, bigger domains of this phase are obtained, and at a coverage close to a saturated monolayer, it is the dominant phase (Figure 7a). In this phase, not all of the molecules look equivalent, resulting in a rectangular unit cell containing four molecules with parameters $b_{1}^{\gamma}=45 \pm 4 \AA$ and $b_{2}^{\gamma}=7.4 \pm 0.5 \AA$, consistent with a commensurate $(12 \sqrt{ } 2 \times 2$ $\sqrt{2)} R 45^{\circ}$ unit cell. The coverage for this phase is then $1 / 12$ $\mathrm{ML}$, larger than that of the $\beta$ phase $(1 / 14 \mathrm{ML})$. The $\gamma$ phase presents long-range order along the $b_{2}^{\gamma}$ direction, while defects appear in the perpendicular direction (linear voids aside the stripes), as indicated by the red arrows in Figure $7 \mathrm{a}$.

This molecular arrangement results from the combination of basic units (building blocks) made of paired molecular stripes. Each stripe is formed by molecules aligned side-by-side with the same in-plane orientation. Each pair consists of one stripe made of molecules oriented transverse to the stripe direction, i.e., at $90^{\circ}$, and the adjacent stripe is made of molecules rotated in-plane at $77^{\circ} \pm 2^{\circ}$. The $\gamma$ domains are formed by the coupling of two pairs of stripes, where the stripes with rotated molecules display an opposite orientation with respect to the stripe direction.

At low coverages, these building blocks can be observed separately, as shown in Figure $7 b, 7 c$, where a three-stripe domain clearly displays the opposite in-plane orientation of TCNQ $\left(\right.$ at $\pm 77^{\circ}$ ) in the stripes aside the central one (TCNQ at $\left.90^{\circ}\right)$. Under certain tip conditions, the filled-state image reveals extra protrusions connecting the molecules of the central stripe with the corresponding ones at the side stripes, which we assign to a signature of $\mathrm{Cu}$ adatoms. A tentative molecular arrangement, commensurate with the underlying $3 \sqrt{2}$ reconstruction, is indicated in the inset of Figure $7 a$, 


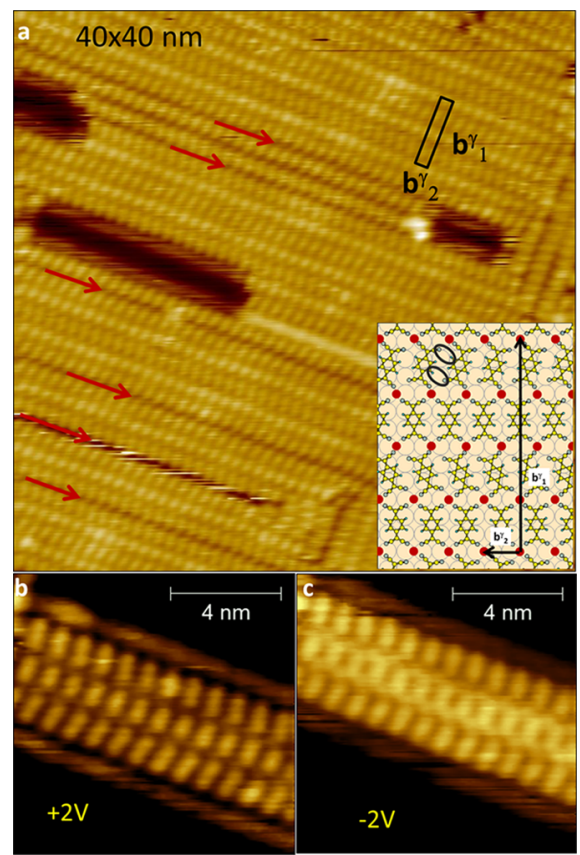

Figure 7. STM images obtained after the adsorption of TCNQ molecules at $420 \mathrm{~K}$ on the $3 \sqrt{ } 2$ surface alloy reconstruction. (a) Empty-state image ( $2 \mathrm{~V}, 5 \mathrm{pA}$ ) of the $\gamma$ phase for a coverage close to $(1 / 12)$ ML. The arrows indicate missing rows (defects). Empty-state (b) and filled-state (c) images of three adjacent stripes of the $\gamma$ phase $(5 \mathrm{pA})$. Inset of (a): proposed model for the $\gamma$ phase on the $3 \sqrt{2}$ reconstruction. The red circles correspond to $\mathrm{Cu}$ adatoms. Only the $\mathrm{Sn}$ atoms (circles) of the substrate are shown.

where all of the $\mathrm{CN}$ groups of the central stripes form coordination bonds. In contrast, the in-plane-rotated molecules have only two of their $\mathrm{CN}$ groups coordinating with $\mathrm{Cu}$ adatoms but they also have $\mathrm{CH} \cdots \mathrm{N}$ bonds with the adjacent molecules. According to this model, each copper atom is coordinated to three $\mathrm{CN}$ groups with a $\mathrm{Cu} / \mathrm{TCNQ}$ stoichiometry ratio $1: 1$. The $\gamma$ phase is thus stoichiometrically equal to the metastable $\alpha$ phase but with the coordination of $\mathrm{Cu}$ adatoms to the nitrile groups changed from 4 to 3 , due to the in-plane rotation of half of the molecules. To accommodate more molecules, the $\mathrm{Cu}$ coordination is changed from 2 ( $\beta$ phase) to 3 ( $\gamma$ phase). A similar behavior was previously observed for $\mathrm{Cu}$ MONs of 1,3,5-tris(pyridyl)benzene on $\mathrm{Au}(111)$, where the $\mathrm{Cu}$ coordination increases for higher molecular densities and was attributed to in-plane compression. ${ }^{48}$

The unit cell of the $\gamma$ phase is too large for a DFT calculation with the substrate. For this reason, to understand the preference of the $\mathrm{Cu}$ atoms for a coordination 3 with the TCNQ molecules, we have performed DFT calculations for two different configurations of unsupported Cu-TCNQ MONs. We have obtained that the preferred configuration corresponds to the one shown in Figure 8a, where the molecules are rotated in-plane, reducing the $\mathrm{Cu}$ coordination from 4 to 3 and forming a $\mathrm{H}$ bond as indicated by the ellipses This configuration results in an important energy gain of 0.26 $\mathrm{eV}$ (Table 1) with respect to a rectangular framework with all of the $\mathrm{Cu}$ atoms coordinating four nitrile groups (Figure $8 \mathrm{~b}$ ). We note that the energy gain is similar to that calculated for a single stripe of TCNQ (without adatoms), when molecules are let to relax by in-plane rotation to form intermolecular $\mathrm{CH}-\mathrm{N}$
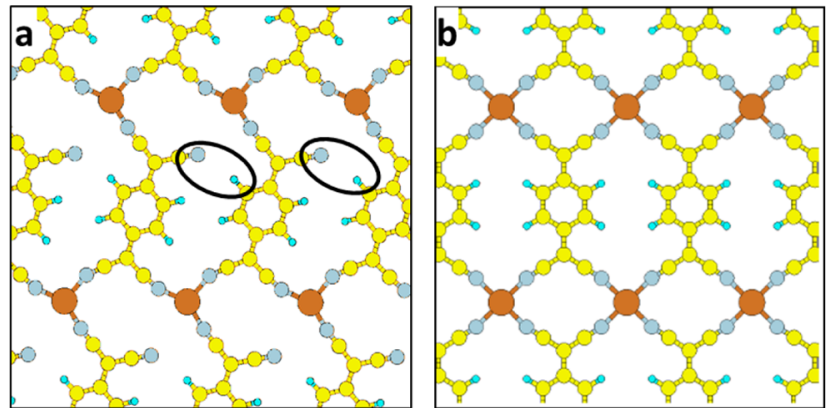

Figure 8. Configurations corresponding to unsupported $\mathrm{Cu}-\mathrm{TCNQ}$ MONs. (a) TCNQ molecules are rotated in-plane to form three short $\mathrm{N}-\mathrm{Cu}$ bonds. (b) Rectangular framework where the $\mathrm{Cu}$ atoms are coordinating four nitrile groups.

bonds (configurations I and II of Figure $5 \mathrm{a}, 5 \mathrm{~b}$ and corresponding energy values in Table 1). This suggests that the preference of TCNQ molecules to adopt the $\gamma$ phase (coordination 3) is mainly associated with the formation of the $\mathrm{H}$ bond.

Information regarding the structure of these phases can be obtained from LEED experiments. Figure $9 a, 9 b$ shows the
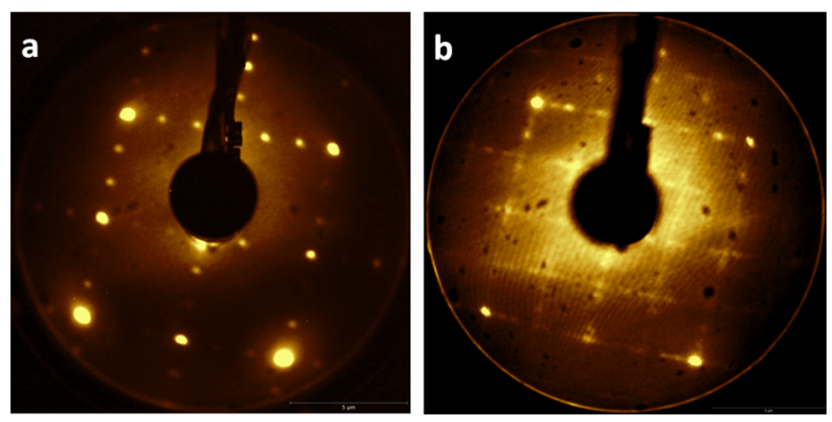

Figure 9. (a) LEED pattern of the bare $3 \sqrt{2}$ surface alloy reconstruction. (b) LEED pattern after the adsorption of TCNQ on the $3 \sqrt{2}$ reconstruction at $420 \mathrm{~K}$ for a coverage similar to the one of Figure $7 \mathrm{a}$. Both patterns were measured at RT and $70 \mathrm{eV}$.

corresponding patterns for the bare $3 \sqrt{ } 2$ reconstruction and for preparation with coverage similar to the one of Figure $7 \mathrm{a}$. Although the $\beta$ and $\gamma$ phases coexist, this coverage is mainly representative of the latter. This pattern corresponds to a $(k \sqrt{ } 2 \times 2 \sqrt{ } 2) R 45^{\circ}$ symmetry and clearly reflects the longrange order observed in the STM images along the $\mathbf{b}_{2}^{\gamma}$ and $\mathbf{b}_{2}^{\beta}$ directions for the $\gamma$ and $\beta$ phases, respectively. In addition, bright spots of the $3 \sqrt{2}$ are clearly seen for the saturated surface, indicating that the underlying $3 \sqrt{2}$ reconstruction is not removed when the adsorption is done at $420 \mathrm{~K}$. This is also supported by XPS measurements of the Sn $4 \mathrm{~d}$ core level, as measured on the bare $3 \sqrt{2}$ surface alloy reconstruction and after the adsorption of TCNQ at $420 \mathrm{~K}$, where the two spectra are practically indistinguishable (see SI-8).

Complementary information on the configuration of the molecular arrangement and the charge transfer can be obtained from polarization-dependent near-edge X-ray absorption fine spectroscopy (NEXAFS). Figure 10 shows the N 1s spectra obtained with $\mathrm{s}$ and $\mathrm{p}$ polarizations after the adsorption of TCNQ on the $3 \sqrt{ } 2$ reconstruction at $420 \mathrm{~K}$ for a coverage similar to the one of Figure 7a (mainly representative of the $\gamma$ phase). For both polarizations, no trace of the LUMO orbital is detected, which should appear at a photon energy of $~ 397.4$ 


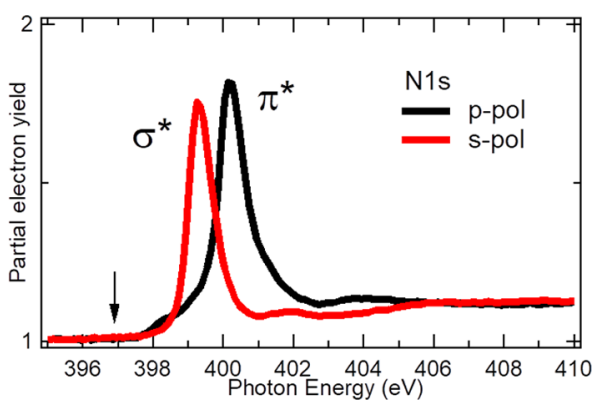

Figure 10. Absorption nitrogen K-edge spectra after the adsorption of TCNQ on the $3 \sqrt{2}$ reconstruction at $420 \mathrm{~K}$ for a coverage similar to the one of Figure $7 \mathrm{a}$.

$\mathrm{eV}^{25,49}$ clearly indicating a charge transfer to the molecule. The two spectra present a main peak at $399.2 \mathrm{eV}$ (s) and at 400.1 $\mathrm{eV}(\mathrm{p})$. These peaks correspond to almost degenerated empty states of the neutral molecule, which are localized on the CN bond of the nitrile group. Two of them are parallel to the $\mathrm{CN}$ bond, while the other two are perpendicular to the $\mathrm{CN}$ bond, producing $\sigma^{*}$ and $\pi^{*}$ resonance in the spectra, respectively. ${ }^{49}$ Notice that we adopt the same notation used in ref 49 for labeling the $\pi^{*}$ and $\sigma^{*}$ LUMOs of the CN groups. The polarization dependence of the $\pi^{*}$ and $\sigma^{*}$ resonances of Figure 10 shows that the molecules in the $\gamma$ phase are adsorbed with $\mathrm{CN}$ almost parallel to the surface. This is in agreement with the small angle $\phi$ theoretically predicted for the unsupported molecular structure depicted in Figure 8a.

Finally, in Figure 11a,11b, we show the N 1s and C 1s XPS spectra, respectively, corresponding to the adsorption of

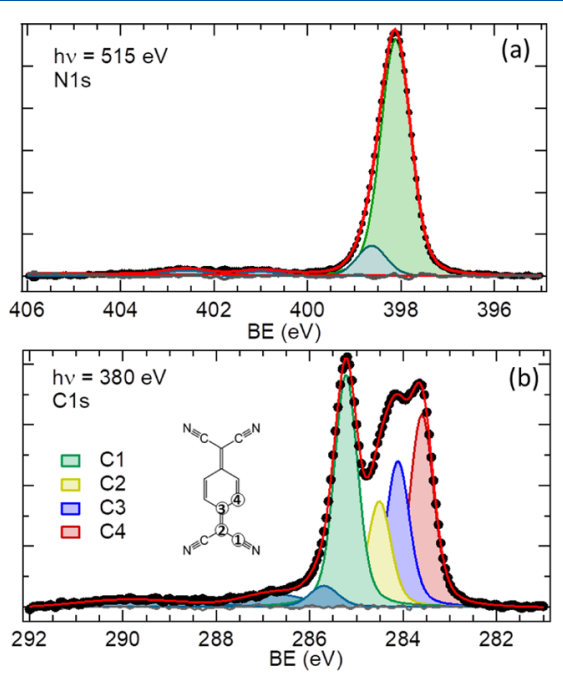

Figure 11. (a) N 1s and (b) C 1s XPS spectra obtained after the adsorption at $420 \mathrm{~K}$ on the $3 \sqrt{2}$ reconstruction for a coverage similar to the one of Figure $7 \mathrm{a}$.

TCNQ on the $3 \sqrt{ } 2$ at $420 \mathrm{~K}$, for a coverage similar to the STM images of Figure $7 \mathrm{a}$. The N 1s core level is fitted with a main component at a binding energy (BE) of $398.1 \mathrm{eV}$ plus three small shake-up peaks. On the other hand, the $C 1$ s peak is fitted with four main components at BEs of 285.20, 284.46, 284.08 , and $283.6 \mathrm{eV}$ and also three small shake-up peaks (see the inset of Figure $11 \mathrm{~b}$ for peak assignments). The relative peak areas are not exactly in their stoichiometry ratio of 4:2:2:4 due to a photoelectron diffraction effect of backward scattering from the substrate atoms. The expected ratio is recovered by increasing the primary photon energy to $800 \mathrm{eV}$, where the weight of photoelectron backward scattering is negligible (see SI-9). We note that the shape of the $C 1$ s spectrum and the performed fitting are comparable to those reported for other MONs based on TCNQ molecules. ${ }^{27,28}$ Finally, the strong attenuation of the shake-up features for both core levels are clear indications that the TCNQ molecules are charged, ${ }^{26-28,50,51}$ in agreement with the NEXAFS measurements.

\section{CONCLUSIONS}

We have studied the adsorption of TCNQ on the $3 \sqrt{2}$ reconstruction of the $\mathrm{SnCu}(001)$ surface alloy.

Our results indicate that the $3 \sqrt{ } 2$ reconstruction is stable upon the adsorption of the strong acceptor TCNQ molecule and generates new 2D Cu-TCNQ structures with unusual geometries and coordination properties.

Surface alloying causes a strong attenuation of the chemical interaction of the molecules with the surface. There are mainly two origins of this effect: (i) the $\mathrm{CN}-\mathrm{Sn}$ interaction is weaker than the $\mathrm{CN}-\mathrm{Cu}$ one and (ii) the $\mathrm{CN}$ groups cannot approach close enough the $\mathrm{Cu}$ atoms underneath. In contrast, surface alloying does not prevent the phenomenon of charge transfer from the surface to the molecules.

On the $\mathrm{SnCu}(100)$ surface alloy, the strong $\mathrm{CN}-\mathrm{Cu}$ interaction drives the formation of different coordination structures with native $\mathrm{Cu}$ adatoms. In addition, the flexible coordination chemistry of $\mathrm{Cu}$ allows the formation of three different phases, namely, $\alpha, \beta$, and $\gamma$, each one with the $\mathrm{Cu}$ ions in a different coordination geometry.

The $\alpha$ phase, with a rectangular structure and 4-fold coordinated $\mathrm{Cu}$ ions, is the less stable one. In fact, the most stable phases are $\beta$ and $\gamma$ ones, with the $\mathrm{Cu}$ ions 2 -fold and 3fold coordinated, respectively. These two phases coexist and give rise to large ribbon-type domains when the molecules are deposited on the surface alloy kept at $420 \mathrm{~K}$. The $\beta$ phase has a TCNQ/Cu ratio of $1: 2\left(\mathrm{Cu}_{2} \mathrm{TCNQ}\right)$, and it is the dominant one at low molecular coverages. In this case, the observed inplane rotation of the molecules is not driven by electrostatic intermolecular interactions ( $\mathrm{H}$ bonds) but by the interaction of the metal center with the alloyed Sn layer. On the other hand, at higher coverages, the $\gamma$ phase (with a TCNQ/Cu ratio of 1:1) dominates, due to its larger molecular density. In this case, two types of supramolecular interactions are combined to stabilized the molecular network: metal-ligand coordination interaction and $\mathrm{H}$ bonds between adjacent molecules. Remarkably, the most stable phases present coordinations 2 and 3 , as previously observed for MONs formed with coinage adatoms and similar strong acceptor molecules Ag-TCNQ ${ }^{20}$ Au-F4TCNQ ${ }^{24}$ and Ag-TCNE. ${ }^{47}$

This work shows that alloying with $\mathrm{Sn}$ is a useful tool to modify the self-assembly of $\mathrm{CN}$-functionalized molecules on $\mathrm{Cu}$ surfaces in a controlled manner. In particular, the $3 \sqrt{2}$ reconstruction can be used as a new low-interacting substrate for the growth of novel MONs with native $(\mathrm{Cu})$ atoms.

\section{ASSOCIATED CONTENT}

\section{Supporting Information}

The Supporting Information is available free of charge at https://pubs.acs.org/doi/10.1021/acs.jpcc.9b08430. 
Complementary information regarding the (a) DFT calculations for a single molecule and single stripe of molecules with and without the substrate (unsupported), (b) commensurate model for the $\beta$ phase; (c) details of the energy barrier calculations for the creation of $\mathrm{Cu}$ adatoms, and (d) XPS spectra of the C 1s and $\mathrm{Sn}$ $4 \mathrm{~d}$ core levels (PDF)

\section{AUTHOR INFORMATION}

\section{Corresponding Authors}

*E-mail: ascolani@cab.cnea.gov.ar (H.A.).

*E-mail: gayone@cab.cnea.gov.ar (J.E.G.).

ORCID $\odot$

A. Verdini: 0000-0001-8880-2080

L. Floreano: 0000-0002-3654-3408

H. Ascolani: 0000-0002-9192-6604

J. E. Gayone: 0000-0003-1484-061X

Notes

The authors declare no competing financial interest.

\section{ACKNOWLEDGMENTS}

We acknowledge financial support from the following Argentine institutions: CONICET (PIP-2015-00274), ANPCYT (PICT-2015-0922) and also from the ICTP-ELETTRA Users Programme. We thank the Centro de Simulación Computacional p/Aplicaciones Tecnológicas (CSC-CONICET) for granting the use of computational resources that allowed us to perform part of the simulations included in this work.

\section{REFERENCES}

(1) Barth, J. V. Molecular Architectonic on Metal Surfaces. Annu. Rev. Phys. Chem. 2007, 58, 375-497.

(2) Klappenberger, F. Two-dimensional functional molecular nanoarchitectures-Complementary investigations with scanning tunneling microscopy and X-ray spectroscopy. Prog. Surf. Sci. 2014, 89, $1-55$.

(3) Gutzler, R.; Stepanow, S.; Grumelli, D.; Lingenfelder, M.; Kern, K. Mimicking Enzymatic Active Sites on Surfaces for Energy Conversion Chemistry. Acc. Chem. Res. 2015, 48, 2132-2139.

(4) Bartels, L. Tailoring molecular layers at metal surfaces. Nat. Chem. 2010, 2, 87-95.

(5) Otero, R.; Gallego, J. M.; de Parga, A. L. V.; Martín, N.; Miranda, R. Molecular Self-Assembly at Solid Surfaces. Adv. Mater. 2011, 23, 5148-5176.

(6) Pussi, K.; AlShamaileh, E.; McLoughlin, E.; Cafolla, A.; Lindroos, M. Determination of the structure of $\mathrm{Cu}\{100\}-\mathrm{p}(3 \sqrt{2} \times$ $\sqrt{ }$ 2)R $45^{\circ}$-Sn by dynamical LEED. Surf. Sci. 2004, 549, 24-30.

(7) Fuhr, J. D.; Gayone, J. E.; Martínez-Blanco, J.; Michel, E. G.; Ascolani, H. Structural and electronic properties of $(3 \sqrt{2} \times \sqrt{2})$ $\mathrm{R} 45^{\circ}-\mathrm{Sn} / \mathrm{Cu}(100)$ : Density functional theory and scanning tunneling microscopy. Phys. Rev. B 2009, 80, No. 115410.

(8) Martínez-Blanco, J.; Joco, V.; Ascolani, H.; Tejeda, A.; Quirós, C.; Panaccione, G.; Balasubramanian, T.; Segovia, P.; Michel, E. G. Phys. Rev. B 2005, 72, No. 041401(R).

(9) Gayone, J. E.; Carrera, A.; Grizzi, O.; Bengió, S.; Sánchez, E. A.; Martínez-Blanco, J.; Michel, E. G.; Fuhr, J. D.; Ascolani, H. Orderdisorder phase transition of vacancies in surfaces: the case of $\mathrm{Sn} /$ $\mathrm{Cu}(001)-0.5$ ML. Phys. Rev. B 2010, 82, No. 035420.

(10) Carrera, A.; Cristina, L. J.; Cossaro, A.; Verdini, A.; Floreano, L.; Fuhr, J.; Gayone, J. E.; Ascolani, H. Controlling Carboxyl Deprotonation on $\mathrm{Cu}(001)$ by Surface Sn Alloying. J. Phys. Chem. C 2013, 117, No. 17058.
(11) Argañaraz, B. Q.; Cristina, L.; Rodríguez, L.; Cossaro, A.; Verdini, A.; Floreano, L.; Fuhr, J.; Gayone, J.; Ascolani, H. Ubiquitous deprotonation of terephthalic acid in the self-assembled phases on $\mathrm{Cu}$ (100). Phys. Chem. Chem. Phys. 2018, 20, 4329-4339.

(12) Fuhr, J. D.; Van Der Meijden, M. W.; Cristina, L. J.; Rodríguez, L. M.; Kellogg, R. M.; Gayone, J. E.; Ascolani, H.; Lingenfelder, M. Chiral expression of adsorbed (MP) 5-amino [6] helicenes: from random structures to dense racemic crystals by surface alloying. Chem. Commun. 2017, 53, 130-133.

(13) Klots, C. E.; Compton, R. N.; Raaen, V. F. Electronic and ionic properties of molecular TTF and TCNQ. J. Chem. Phys. 1974, 60, $1177-1178$.

(14) Torrente, I. F.; Franke, K. J.; Pascual, J. I. Structure and electronic configuration of tetracyanoquinodimethane layers on a $\mathrm{Au}$ (111) surface. Int. J. Mass Spectrom. 2008, 277, 269-273.

(15) Tseng, T.-C.; Urban, C.; Wang, Y.; Otero, R.; Tait, S. L.; Alcamí, M.; Écija, D.; Trelka, M.; Gallego, J. M.; Lin, N.; et al. Charge-transfer-induced structural rearrangements at both sides of organic/metal interfaces. Nat. Chem. 2010, 2, 374-379.

(16) Barja, S.; Garnica, M.; Hinarejos, J.; de Parga, A. V.; Martin, N.; Miranda, R. Self-organization of electron acceptor molecules on graphene. Chem. Commun. 2010, 46, 8198-8200.

(17) Garnica, M.; Stradi, D.; Barja, S.; Calleja, F.; Díaz, C.; Alcamí, M.; Martin, N.; de Parga, A. V.; Martin, F.; Miranda, R. Long-range magnetic order in a purely organic $2 \mathrm{D}$ layer adsorbed on epitaxial graphene. Nat. Phys. 2013, 9, 368-374.

(18) Park, C.; Rojas, G. A.; Jeon, S.; Kelly, S. J.; Smith, S. C.; Sumpter, B. G.; Yoon, M.; Maksymovych, P. Weak competing interactions control assembly of strongly bonded TCNQ ionic acceptor molecules on silver surfaces. Phys. Rev. B 2014, 90, No. 125432.

(19) Stradi, D.; Borca, B.; Barja, S.; Garnica, M.; Diaz, C.; Rodriguez-Garcia, J. M.; Alcami, M.; Vazquez de Parga, A. L.; Miranda, R.; Martin, F. Understanding the self-assembly of TCNQ on $\mathrm{Cu}(111)$ : a combined study based on scanning tunnelling microscopy experiments and density functional theory simulations. RSC Adv. 2016, 6, 15071-15079.

(20) Blowey, P.; Velari, S.; Rochford, L.; Duncan, D.; Warr, D.; Lee, T.-L.; De Vita, A.; Costantini, G.; Woodruff, D. Re-evaluating how charge transfer modifies the conformation of adsorbed molecules. Nanoscale 2018, 10, 14984-14992.

(21) Tseng, T.-C.; Lin, C.; Shi, X.; Tait, S. L.; Liu, X.; Starke, U.; Lin, N.; Zhang, R.; Minot, C.; Hove, M. A. V.; et al. Two-dimensional metal-organic coordination networks of $\mathrm{Mn}-7,7,8,8$-tetracyanoquinodimethane assembled on $\mathrm{Cu}(100)$ : Structural, electronic, and magnetic properties. Phys. Rev. B 2009, 80, No. 155458.

(22) Abdurakhmanova, N.; Floris, A.; Tseng, T.-C.; Comisso, A.; Stepanow, S.; De Vita, A.; Kern, K. Stereoselectivity and electrostatics in charge-transfer Mn-and Cs-TCNQ 4 networks on Ag (100). Nat. Commun. 2012, 3, No. 940.

(23) Tseng, T.-C.; Abdurakhmanova, N.; Stepanow, S.; Kern, K. Hierarchical Assembly and Reticulation of Two-Dimensional Mnand $\mathrm{Ni}-\mathrm{TCNQ}_{\mathrm{x}}(\mathrm{x}=1,2,4)$ Coordination Structures on a Metal Surface. J. Phys. Chem. C 2011, 115, 10211-10217.

(24) Faraggi, M. N.; Jiang, N.; Gonzalez-Lakunza, N.; Langner, A.; Stepanow, S.; Kern, K.; Arnau, A. Bonding and charge transfer in metal-organic coordination networks on $\mathrm{Au}$ (111) with strong acceptor molecules. J. Phys. Chem. C 2012, 116, 24558-24565.

(25) Abdurakhmanova, N.; Tseng, T.-C.; Langner, A.; Kley, C. S.; Sessi, V.; Stepanow, S.; Kern, K. Superexchange-mediated ferromagnetic coupling in two-dimensional Ni-TCNQ networks on metal surfaces. Phys. Rev. Lett. 2013, 110, No. 027202.

(26) Wäckerlin, C.; Iacovita, C.; Chylarecka, D.; Fesser, P.; Jung, T. A.; Ballav, N. Assembly of $2 \mathrm{D}$ ionic layers by reaction of alkali halides with the organic electrophile 7,7,8,8-tetracyano-p-quinodimethane (TCNQ). Chem. Commun. 2011, 47, 9146-9148.

(27) Blowey, P. J.; Rochford, L. A.; Duncan, D. A.; Warr, D.; Lee, T.L.; Woodruff, D.; Costantini, G. Probing the interplay between 
geometric and electronic structure in a two-dimensional K-TCNQ charge transfer network. Faraday Discuss. 2017, 204, 97-110.

(28) Rodríguez, L. M.; Fuhr, J. D.; Machaín, P.; Ascolani, H.; Lingenfelder, M.; Gayone, J. E. Building two-dimensional metalorganic networks with tin. Chem. Commun. 2019, 55, 345-348.

(29) Pawin, G.; Wong, K. L.; Kim, D.; Sun, D.; Bartels, L.; Hong, S.; Rahman, T. S.; Carp, R.; Marsella, M. A Surface Coordination Network Based on Substrate-Derived Metal Adatoms with Local Charge Excess. Angew. Chem., Int. Ed. 2008, 47, 8442-8445.

(30) Pivetta, M.; Pacchioni, G. E.; Fernandes, E.; Brune, H. Temperature-dependent self-assembly of NC-Ph5-CN molecules on $\mathrm{Cu}(111)$. J. Chem. Phys. 2015, 142, No. 101928.

(31) Shchyrba, A.; Nguyen, M.-T.; Wackerlin, C.; Martens, S.; Nowakowska, S.; Ivas, T.; Roose, J.; Nijs, T.; Boz, S.; Schar, M.; et al. Chirality transfer in 1D self-assemblies: influence of H-bonding vs metal coordination between dicyano [7] helicene enantiomers. J. Am. Chem. Soc. 2013, 135, 15270-15273.

(32) Sirtl, T.; Schlogl, S.; Rastgoo-Lahrood, A.; Jelic, J.; Neogi, S.; Schmittel, M.; Heckl, W. M.; Reuter, K.; Lackinger, M. Control of intermolecular bonds by deposition rates at room temperature: hydrogen bonds versus metal coordination in trinitrile monolayers. J. Am. Chem. Soc. 2013, 135, 691-695.

(33) Müller, K.; Moreno-López, J. C.; Gottardi, S.; Meinhardt, U.; Yildirim, H.; Kara, A.; Kivala, M.; Stöhr, M. Cyano-Functionalized Triarylamines on Coinage Metal Surfaces: Interplay of Intermolecular and Molecule-Substrate Interactions. Chem. - Eur. J. 2016, 22, 581589.

(34) Gotter, R.; Ruocco, A.; Morgante, A.; Cvetko, D.; Floreano, L.; Tommasini, F.; Stefani, G. The ALOISA end station at Elettra:: a novel multicoincidence spectrometer for angle resolved APECS. Nucl. Instrum. Methods Phys. Res., Sect. A 2001, 467, 1468-1472.

(35) Floreano, L.; Naletto, G.; Cvetko, D.; Gotter, R.; Malvezzi, M.; Marassi, L.; Morgante, A.; Santaniello, A.; Verdini, A.; Tommasini, F.; et al. Performance of the grating-crystal monochromator of the ALOISA beamline at the Elettra Synchrotron. Rev. Sci. Instrum. 1999, $70,3855-3865$.

(36) Floreano, L.; Cossaro, A.; Gotter, R.; Verdini, A.; Bavdek, G.; Evangelista, F.; Ruocco, A.; Morgante, A.; Cvetko, D. Periodic arrays of Cu-phthalocyanine chains on Au (110). J. Phys. Chem. C 2008, 112, No. 10794.

(37) Giannozzi, P.; Baroni, S.; Bonini, N.; Calandra, M.; Car, R.; Cavazzoni, C.; Ceresoli, D.; Chiarotti, G. L.; Cococcioni, M.; Dabo, I.; et al. QUANTUM ESPRESSO: a modular and open-source software project for quantum simulations of materials. J. Phys.: Condens. Matter 2009, 21, No. 395502.

(38) Sabatini, R.; Gorni, T.; de Gironcoli, S. Nonlocal van der Waals density functional made simple and efficient. Phys. Rev. B 2013, 87, No. 041108.

(39) Vydrov, O. A.; Voorhis, T. V. Nonlocal van der Waals density functional: The simpler the better. J. Chem. Phys. 2010, 133, No. 244103.

(40) González-Moreno, R.; Sánchez-Sánchez, C.; Trelka, M.; Otero, R.; Cossaro, A.; Verdini, A.; Floreano, L.; Ruiz-Bermejo, M.; GarcíaLekue, A.; Martín-Gago, J. Á.; et al. Following the metalation process of protoporphyrin IX with metal substrate atoms at room temperature. J. Phys. Chem. C 2011, 115, 6849-6854.

(41) Lovat, G.; Forrer, D.; Abadia, M.; Dominguez, M.; Casarin, M.; Rogero, C.; Vittadini, A.; Floreano, L. On-surface synthesis of a pure and long-range-ordered titanium (IV)-porphyrin contact layer on titanium dioxide. J. Phys. Chem. C 2017, 121, 13738-13746.

(42) Scheffler, M.; Stampfl, C. In Handbook of Surface Science, Vol. 2: Electronic Structure; Horn, K., Scheffler, M. Elsevier: Amsterdam, 2000; Chapter 5, pp 285-356.

(43) Bader, R. F. W. Encyclopedia of Computational Chemistry; John Wiley \& Sons, Ltd, 2002.

(44) Henkelman, G.; Arnaldsson, A.; Jonsson, H. A fast and robust algorithm for Bader decomposition of charge density. Comput. Mater. Sci. 2006, 36, 354-360.
(45) Bischoff, F.; He, Y.; Seufert, K.; Stassen, D.; Bonifazi, D.; Barth, J. V.; Auwarter, W. Tailoring Large Pores of Porphyrin Networks on $\mathrm{Ag}(111)$ by Metal-Organic Coordination. Chem. - Eur. J. 2016, 22, $15298-15306$.

(46) Nilsson, A.; Pettersson, L.; Hammer, B.; Bligaard, T.; Christensen, C. H.; Nørskov, J. K. The electronic structure effect in heterogeneous catalysis. Catal. Lett. 2005, 100, 111-114.

(47) Rodríguez-Fernández, J.; Lauwaet, K.; Herranz, M. Á.; Martín, N.; Gallego, J. M.; Miranda, R.; Otero, R. Temperature-controlled metal/ligand stoichiometric ratio in Ag-TCNE coordination networks. J. Chem. Phys. 2015, 142, No. 101930.

(48) Liu, J.; Lin, T.; Shi, Z.; Xia, F.; Dong, L.; Liu, P. N.; Lin, N. Structural transformation of two-dimensional metal-organic coordination networks driven by intrinsic in-plane compression. J. Am. Chem. Soc. 2011, 133, 18760-18766.

(49) Fraxedas, J.; Lee, Y. J.; Jiménez, I.; Gago, R.; Nieminen, R. M.; Ordejón, P.; Canadell, E. occupied states of TTF-TCNQ by XANES and first-principles calculations. Phys. Rev. B 2003, 68, No. 195115.

(50) Lindquist, J. M.; Hemminger, J. C. High energy resolution x-ray photoelectron spectroscopy studies of tetracyanoquinodimethane charge transfer complexes with copper, nickel, and lithium. Chem. Mater. 1989, 1, 72-78.

(51) Precht, R.; Hausbrand, R.; Jaegermann, W. Electronic structure and electrode properties of tetracyanoquinodimethane (TCNQ): a Surf. Sci. investigation of lithium intercalation into TCNQ. Phys. Chem. Chem. Phys. 2015, 17, 6588-6596. 